\title{
ORGANIC MATERIAL AND GASES IN THE EARLY SOLAR SYSTEM: THE METEORITE RECORD
}

\author{
L. Remusat ${ }^{1}$
}

\begin{abstract}
The most primitive chondrites and comets have undergone few modifications during the 4.56 billion years of the Solar System and can deliver to laboratories the components that witnessed the origin of the protosolar nebula. These components include organics, water and other volatile components that were formed in the parent molecular cloud of the solar system or during the protosolar epoch. This paper focuses on organic matter, water and noble gases contained in carbonaceous chondrites with a special emphasis on opened questions that could be assessed by laboratory experiments in order to shed new light on the origin of the solar system materials and planets.
\end{abstract}

\section{Introduction}

The Solar System formed from a dusty molecular cloud 4.56 billion years ago. During its first ages, the condensation of materials resulted in the formation of solids that hereafter accreted to form planet embryos. These solids have accreted ices, organic grains and silicate particles recording the signature of the processes occurring during the birth of the Solar System. Chondrites are undifferentiated meteorites and are considered as primitive objects in the solar system, along with micrometeorites, interplanetary dust particles (IDPs) and cometary grains. They result from the disruption of small bodies that did not undergo differentiation, i.e. internal heating was not enough to induce the formation of layers with different mineralogical and chemical composition by melting/crystallization in contrast to terrestrial planets, like the Earth.

Several classes of chondrites are defined depending on their chemical and mineralogical composition. Carbonaceous chondrites are the oldest meteorites in the solar system (Amelin et al. 2002) and their elemental composition is close to the composition of the Sun, except for the most volatile elements like H, He, C, O and N (Anders et al. 1989). They are considered to reflect the composition of the

1 Laboratoire de Minéralogie et Cosmochimie du Muséum, UMR CNRS 7202, Muséum National d'Histoire Naturelle, CP. 52, 57 rue Cuvier, 75231 Paris Cedex 05, France 
molecular cloud that gave birth to the solar system (Lodders 2003). As they have the highest content in organic matter, water and noble gases, the present paper will describe these three components in carbonaceous chondrites, which represent $4 \%$ of the meteorites found (including falls and findings) on Earth.

However, some chemical reactions have occurred since the chondrites accretion. The chondrites parent bodies (i.e. the parent asteroids) accreted significant amount of short live radionucleides, like for instance ${ }^{26} \mathrm{Al}$. The decay of these isotopes has resulted in some modifications of the accreted components by hydrothermal (aqueous) alteration or thermal metamorphism on the parent body (Brearley 2003; Bunch \& Chang 1980). Extends of these modifications are variable among chondrites class depending on the size/composition of their parent body (Weisberg et al. 2006; Kallemeyn et al. 1981). CI are the most hydrated carbonaceous chondrites, CM are less hydrated. CR are often considered as the least modified carbonaceous chondrites on their parent bodies, with the less processed materials. They are supposed to have been subjected to mild metamorphism and hydrothermalism. $\mathrm{CO}$ and $\mathrm{CV}$ are the metamorphosed counter parts of the CM. They differ by their content in refractory elements and the size of their chondrules (both being larger in CV). They have been subject to temperatures higher than $350{ }^{\circ} \mathrm{C}$, and contain much less organics and water.

The goal of this paper is to provide a brief review of the content in the volatile components in carbonaceous chondrites, in order to shed light on a few questions that arise from the study of these objects and could be assessed by combining advances in cosmochemistry and astrophysics.

\section{How did the organics in carbonacenous chondrites form?}

Carbonaceous chondrites contain up to $5 \mathrm{wt} \%$ of organic matter occuring as soluble compounds and macromolecular insoluble organic matter (IOM). It is found in the matrix of chondrites, associated with phyllosilicates (Pearson et al. 2002). Organics likely accreted with other volatiles components in the matrix. This is confirmed by fluorescence microscopy (Alpern \& Benkheiri 1973), which shows that organic matter occurs as circular particles, evenly distributed in the matrix of hydrated chondrites and with a diameter around a couple of microns. Organic matter has also been observed to form nanoglobules (Nakamura et al. 2002; Garvie \& Buseck 2004); analogues were produced during laboratory experiment intending to reproduce ejecta gas from evolved stars (Saito \& Kimura 2009).

\subsection{Molecular structure of the IOM: A peculiar macromolecule}

The molecular model (Derenne \& Robert 2010) presented in Figure 1 summarizes the results of 20 years of molecular analysis of Murchison IOM by spectroscopic techniques (like infrared spectrometry or solid state NMR) or thermal and chemical degradation (pyrolysis or oxidations). IOM is constituted by small aromatic units with numerous substitutions by short and branched aliphatic chains; this results in a high degree of cross-linking. In CI and CM, IOM contains organic radicals 
heterogeneously distributed (leading to the occurrence of radical rich clusters Binet et al. 2002) and abundant diradicaloids (unpaired radicals). The later are not detected in any other natural organic matter (Binet et al. 2004). Their occurrence may likely indicate that radical chemistry played a key role in the synthesis of chondritic IOM during a preaccretion event.

The effects of parent body processes are noticeable. Thermal metamorphism will induce dramatic modifications of the carbon skeleton through carbonization followed by graphitization (Rouzaud \& Oberlin 1989). As shown by Raman spectrometry, the structural order of the carbonaceous matter increases with temperature (Bonal et al. 2006). There is also a clear trend in losing the heteroelements (evidenced by a decrease in $\mathrm{H} / \mathrm{C}, \mathrm{N} / \mathrm{C}$ and $\mathrm{O} / \mathrm{C}$ elemental ratios) a loss of aliphatic chains and in increasing the size of aromatic units (Remusat et al. 2008). On the other hand, hydrothermal alteration may induce oxidation of some functional groups like $\mathrm{O}$ and $\mathrm{S}$ containing groups. In the IOM, oxidized moieties like ether and ester groups are more abundant in more altered carbonaceous chondrites (Remusat et al. 2005). The S-containing groups show a higher degree of oxidation in the more altered carbonaceous chondrites (Orthous-Daunay et al. 2010).

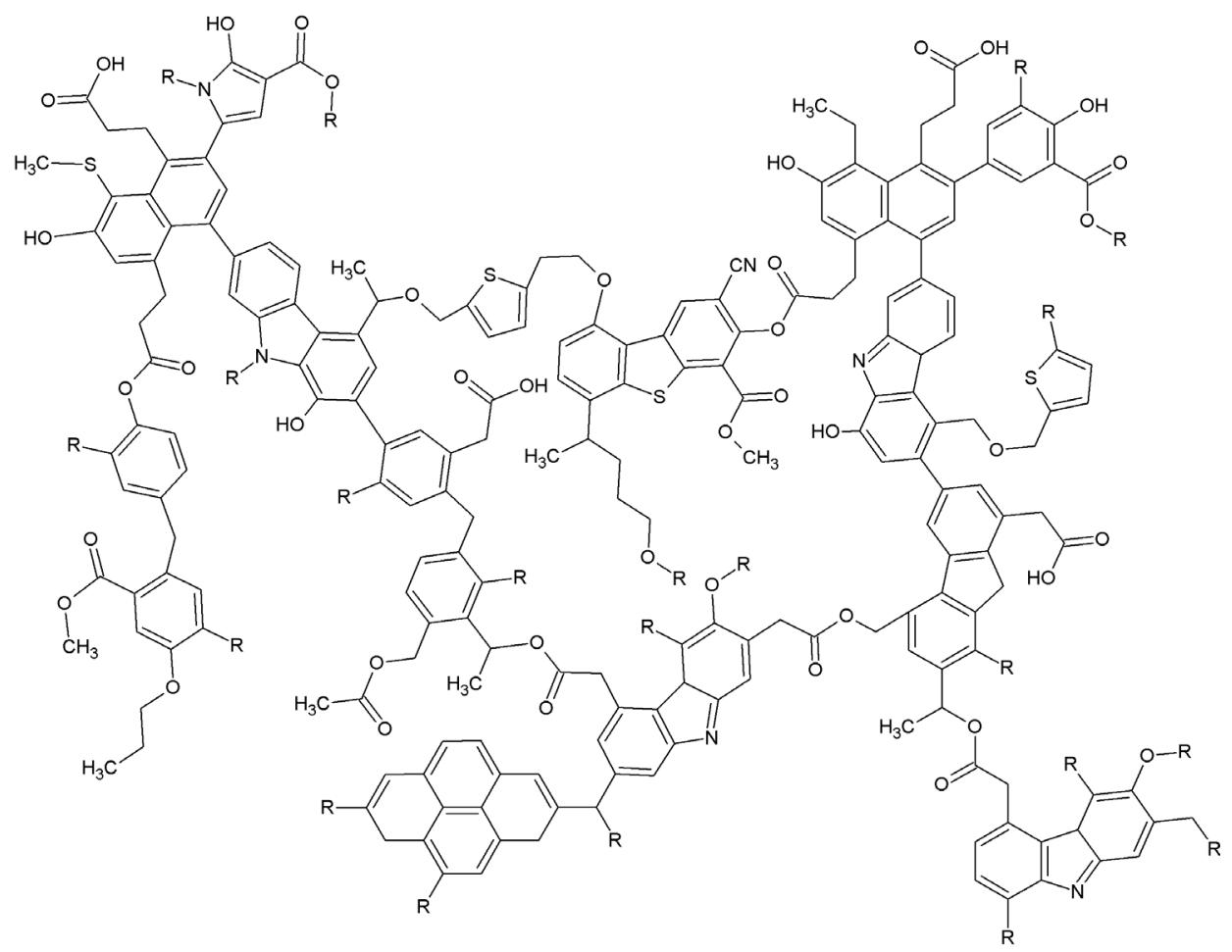

Fig. 1. Molecular model of Murchison IOM. R means an organic moiety. From Derenne \& Robert (2010), modified. 
All these studies indicate that the molecular structure of the IOM in carbonaceous chondrites can not be readily compared with models proposed for complex organic molecules in space (ISM or protosolar disks) based on observations (Pendleton \& Allamandola 2002) or laboratory experiments (Dartois et al. 2005). However, the carbon skeleton of less heated chondrites (CI, CM and CR) should not be significantly altered since the accretion of the parent body, as the molecular structure of their IOMs seems to be broadly similar. The question of the formation of the IOM in carbonaceous chondrites is still unresolved. Its structure has not yet been reproduced in laboratory analogues and we still do not know if it is the result of photochemistry in ices, or chemistry in the gas phase or thermal processing of grains mantle.

\subsection{Soluble organic compounds}

Carbonaceous chondrites contain a diverse suite of soluble organic compounds as revealed by the study of the Murchison meteorite (Pizzarello et al. 2006). The most abundant soluble compounds in Murchison are carboxylic acids (330 ppm Yuen \& Kvenvolden 1973; Krishnamurthy et al. 1992). Among all the other compounds, Murchison contains several groups of compounds of biological interest: amino acids (up to 60 ppm, Kvenvolden et al. 1970), sugar (Cooper et al. 2001), carboxamides (Cooper \& Cronin 1995) and N-heterocycles (Hayatsu et al. 1975). These soluble compounds share several molecular properties (Pizzarello et al. 2006): 1- they show a complete structural diversity (for each formula, every isomer is detected), 2 - the abundance decreases when the carbon number increases and in general 3- branched chains are more abundant. Enantiomeric excesses have been detected in chondrites, in amino acids (Engel \& Nagy 1982; Glavin et al. 2009). These excesses have been suggested to result from UV circularly polarized light irradiation (Rubenstein et al. 1983) or reactions involving chiral mineral surfaces (Glavin et al. 2009).

The soluble compounds exhibit a complex isotopic distribution pointing to a complex origin (Gilmour et al. 2003). The soluble fraction is likely a mixing between moieties that could have been formed on the parent body, from the reaction of precursors that could have been accreted in ices or from the hydrolysis of the IOM, and compounds that could have been synthesized prior to accretion and preserved trapped in the matrix of the carbonaceous chondrites.

The question of the genetic link between soluble and insoluble organic matter is still debated; the IOM could for instance release some soluble compounds during hydrothermal alteration (Sephton et al. 1998; Yabuta et al. 2007). To date, only aromatic hydrocarbons could be related to the macromolecular carbon (Sephton et al. 1998).

\section{How did the asteroids trap noble gases?}

Noble gases occur as very low concentration in meteorites. For instance, an abundance of 0.6 ppt (part per trillion) of Xenon is considered as pretty high. Several 
origins are possible for noble gases in chondrites (Podosek et al. 2003; Wieler et al. 2006): radioactive decay (for instance ${ }^{129} \mathrm{Xe}$ is produced by radioactive decay of $\left.{ }^{129} \mathrm{I}\right)$, implantation from solar wind, production by nuclear effects like spallation or accreted in carrier phases. Then two distinct components can be distinguished: the solar (mainly implanted) and the planetary noble gases (trapped in carrier phases and often considered as primordial). An interesting property of some noble gases is that they have several isotopes (for instance 9 for $\mathrm{Xe}$ ), allowing us to test possible mechanisms for their origin.

Elemental and isotopic patterns have been determined for noble gases (Fig. 2). Some carrier phases are presolar, like $\mathrm{SiC}$ or nano diamonds and their isotopic pattern can be used as a fingerprint of the origin of the carrier phase but also to determine its abundance. Some components, like HL, are named anomalous components as they require nucleosynthetic processes (i.e. presolar phenomena), in addition to common physical and chemical processes (like diffusion or condensation), to account for the observed isotopic pattern.

One reservoir, the P1 component, also called Q phase (for quintessence) was defined by Lewis et al. (1975) based on its resistance to $\mathrm{HF} / \mathrm{HCl}$ leaching and disruption in $\mathrm{HNO}_{3}$. It seems to be a main reservoir of primordial noble gases, and it is not yet fully identified (Busemann et al. 2000). Some striking observations have been made on Q noble gases: 1- it releases most of its noble gases at $1000{ }^{\circ} \mathrm{C}$, and this temperature is the same for all the noble gases (Huss et al. 1996); 2- the relative abundance of each noble gas is the same in all the chondrites (Busemann et al. 2000). The exact process that trapped the noble gases in Q is also unknown. It was suggested that the texture of the IOM could trap noble gases atoms in holes in the aromatic and aliphatic network of the IOM (Marrocchi et al. 2005) or that noble gases could be adsorbed onto defects of organic surfaces (Marrocchi et al. 2011).
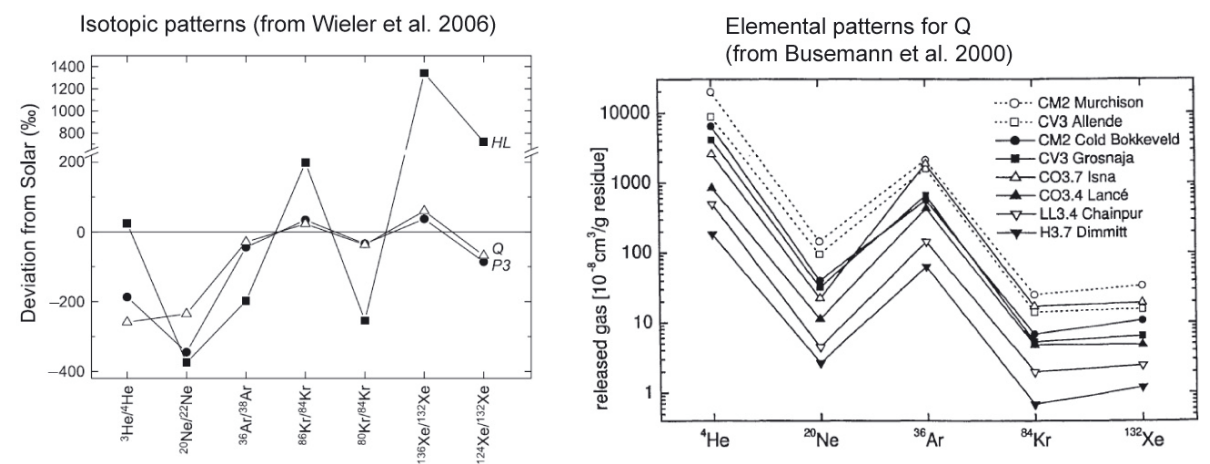

Fig. 2. Noble gases in carbonaceous chondrites. Several reservoirs can be distinguished thanks to their isotopic patterns (left plot). Some carrier phase were identified, like presolar nanodiamonds for HL and P3, by etching experiments. Q shows similar elemental pattern in different chondrites having very different parent body histories and bulk compositions (right plot). 
A lot of questions remain opened about the accretion and origin of noble gases in chondrites like how they got fractionated, how can we explain that the same pattern is observed in several classes of carbonaceous exhibiting different parent body histories or how they got trapped in solids. The framework is complicated by the superimposition of signatures of presolar and solar components in addition to parent body processes.

\section{How can we interpret the encrypted isotopic record of water and organics in chondrites?}

With the exception of presolar grains, characterised by isotopic compositions that can only be explained by nuclear processes in supernovae or evolved stars, most of chondrites components are thought to have formed during the early stages of the Solar System (in the so called protosolar nebula) or in the parent molecular cloud that evolved into our solar system. Despite numerous measurements, interpretations of the isotopic composition of organics and hydrated minerals in chondrites are still a matter of intense debate. It must be noted that in all the hydrated chondrites, water is always depleted in D compared to organic matter, whereas the equilibrium requires the opposite, whatever the location of the $\mathrm{H}$ in the molecule (Sessions et al. 2004; Remusat et al. 2010). This indicates that isotopic disequilibrium has been preserved during 4.56 billion years on the parent body of these carbonaceous chondrites and that parent body processes may not have erased the signatures of the synthesis.

\subsection{Water in chondrites}

In contrast to comets, meteorites do not contain any ice or liquid water. Water has reacted with minerals and produced phyllosilicates and other secondary phases in altered carbonaceous chondrites (Brearley 2006) on the parent body (DuFresne \& Anders 1962; Bunch \& Chang 1980) or during a pre-accretion process (Bischoff 1998). The water content in CM and CR goes from 3 to $14 \mathrm{wt} \%$, it goes up to $15 \mathrm{wt} \%$ in CI (Alexander et al. 2010). This water could have accreted as ice mixed with silicates grains or was trapped in phyllosilicates.

As seen in comets (Hartogh et al. 2011) the D/H ratio of water in chondrites is variable and may be influenced by parent body processes (Alexander et al. 2010) and by pre accretion conditions, like temperature gradients in the protosolar nebula (Robert 2002). Meteoritic water has an average value around the terrestrial ratio, i.e. 6 times more enriched in $\mathrm{D}$ than the protosolar molecular hydrogen, but with some variations. In CI and CM, water is comprised within the range $-180 \%$ < $\delta \mathrm{D}<+30 \%$ (i.e. $128 \times 10^{-6}<\mathrm{D} / \mathrm{H}<160 \times 10^{-6}$ ), but is heavier in CR chondrites: $\delta \mathrm{D}=680 \%$; D $/ \mathrm{H}=262 \times 10^{-6}$ (Robert \& Epstein 1982; Robert 2002; Eiler \& Kitchen 2004; Alexander et al. 2010). 


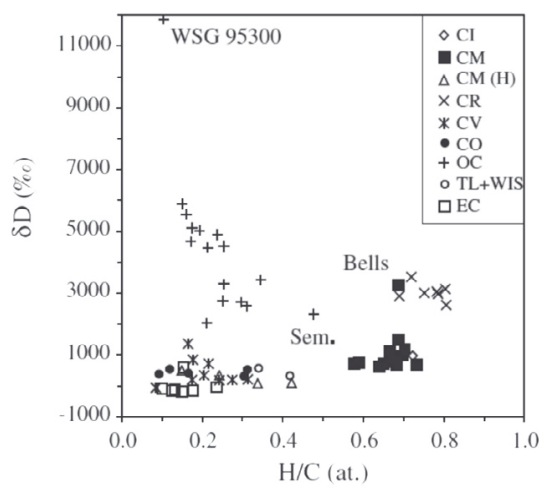

Fig. 3. $\mathrm{H}$ isotopic compositions vs. the $\mathrm{H} / \mathrm{C}$ ratio in the IOM of chondrites of various classes, from Alexander et al. (2010). OC are ordinary chondrites, EC are enstatite chondrites, all the others are carbonaceous chondrites.

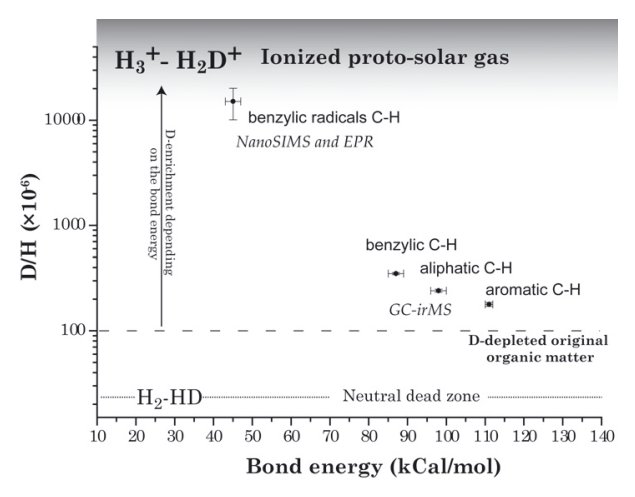

Fig. 4. D/H isotopic ratio versus $\mathrm{C}-\mathrm{H}$ bond energy among different molecular components in Orgueil IOM. The observed trend was interpreted as the result of an isotopic exchange reaction between D-poor organics and D-rich gaseous molecules like $\mathrm{H}_{2} \mathrm{D}^{+}$or $\mathrm{HD}_{2}^{+}$(Remusat et al. 2006).

\section{2 $\mathrm{D} / \mathrm{H}$ ratio heterogeneities in the IOM}

Observations. Figure 3 shows the variations between the different classes of chondrites. In chondritic IOMs, $\delta \mathrm{D}$ ranges from $-100 \%$ in enstatite chondrites up to $11000 \%$ in some ordinary chondrites (Alexander et al. 2010). CI and CM, the most aqueously altered carbonaceous chondrites, exhibit similar ratios with the exception of Bells CM chondrite. CR chondrites, believed to be the less modified carbonaceous chondrites have a $\mathrm{D} / \mathrm{H}$ ratio twice as large; their IOM is often assumed to be the most pristine. CO and oxidized CV, heated carbonaceous chondrites, have $\mathrm{D} / \mathrm{H}$ ratio around the terrestrial ratio; they are depleted compared to CI, CM and CR (2 times less compared to CM and 4 times less than CR). In these groups, the $\mathrm{D} / \mathrm{H}$ ratio does not correlate with an evolution in the $\mathrm{H} / \mathrm{C}$ ratio. Ordinary chondrites exhibit a negative trend in the diagram, with the samples with the lowest $\mathrm{H} / \mathrm{C}$ having the highest $\mathrm{D}$-enrichment. The reduced $\mathrm{CV}$ also exhibit the same trend, though the range of $\mathrm{D} / \mathrm{H}$ ratio is much smaller (a few hundred of permil compared to several thousands of permil in delta units). It must be noted that

The isotopic compositions may be expressed in delta units, using:

$$
\delta(\%)=\left(\frac{R_{\text {sample }}}{R_{\text {standard }}}-1\right) \times 1000
$$

with $R_{\text {sample }}$ being the sample isotopic ratio and $R_{\text {standard }}$ the ratio of a terrestrial standard. For $\mathrm{H}$ isotopes, we use: Standard Mean Ocean Water $\left(S M O W: D / H=155.76 \times 10^{-6}\right)$. 
the lowest isotopic enrichment was measured in Abee enstatite chondrite $(\delta \mathrm{D}=$ $-480 \%$; D $/ \mathrm{H}=80 \times 10^{-6}$ Yang \& Epstein 1983).

Isotopic imaging has revealed that $\mathrm{H}$ isotopes can exhibit large variations in the IOM of CI, CM and CR chondrites with the occurrence of D-rich "hot spots": micron sized areas with large positive $\mathrm{D}$ anomalies, which could go up to $\delta \mathrm{D}=$ $8000 \%\left(\mathrm{D} / \mathrm{H}=1402 \times 10^{-6}\right.$; Busemann et al. 2006; Nakamura-Messenger et al. 2006; Remusat et al. 2009). No D-rich hot spots are observed in IOM of heated chondrites, i.e. CO, CV (Remusat et al. 2009) pointing out to their thermal sensitivity.

The distribution of the deuterium has also been studied at the molecular scale (Wang et al. 2005; Remusat et al. 2006; Huang et al. 2007). A relation between the $\mathrm{C}-\mathrm{H}$ bond dissociation energy and the $\mathrm{D} / \mathrm{H}$ ratio has been revealed in Orgueil IOM (Fig. 4; Remusat et al. 2006). In addition, radicals in the IOM of Orgueil are D-rich: $\mathrm{D} / \mathrm{H}=15000 \pm 5000 \times 10^{-6} ; \delta \mathrm{D}=95300 \%$ (Gourier et al. 2008). Only these moieties have D-enrichment comparable to the highest enrichments observed in some organics in the ISM (45000 to $60000 \times 10^{-6}$; Millar 2003). Although the pattern of Figure 4 has been reproduced experimentally (Robert et al. 2011), more laboratory experiments are required to determine kinetic parameters or fractionation factors in order to fully constrain the involved processes.

In summary, organics in chondrites are more D-rich than the protosolar $\mathrm{H}_{2}$, with $\mathrm{D} / \mathrm{H}$ ratios ranging from 5 to 20 times the protosolar $\mathrm{D} / \mathrm{H}$ (with a $\mathrm{D} / \mathrm{H}=$ $25 \times 10^{-6}, \delta \mathrm{D}=-840 \%$, as determined from the Jupiter atmosphere and solar wind, Geiss \& Gloecker 1998). Moreover the D is heterogeneously distributed between and within the samples, at the micron and the molecular scales. The interpretation of the $\mathrm{D} / \mathrm{H}$ ratio in the IOMs of carbonaceous chondrites remains very speculative: it has been attributed to interstellar (Robert \& Epstein 1982; Yang \& Epstein 1983), nebular (Remusat et al. 2006) or parent body processes (Alexander et al. 2010)! This issue results from the superimposition of synthetis reactions (primary process) and the following modifications (secondary processes) that could affect the isotopic record of IOM in the gas phase or on the parent body.

Possible interpretations. The D-enrichment in chondritic organics in chondrites was interpreted as an interstellar origin, as large D-enrichments are predicted and observed in the ISM (Sandford et al. 2001; Millar 2003). However, an interstellar origin of the $\mathrm{D} / \mathrm{H}$ ratio would require the precursor to survive and escape isotopic exchange during the ash conditions experienced in the ISM before the formation of the solar system or in the protosolar nebula during its early ages. From laboratory experiments (Muñoz Caro et al. 2006), it has been shown that irradiations occuring in the ISM would likely erase a D-rich signature and lower to $\mathrm{D} / \mathrm{H}$ ratio due to exchange with the molecular $\mathrm{H}_{2}$ gas (with a $\mathrm{D} / \mathrm{H}$ ratio close to $\left.16 \times 10^{-6}\right)$. Then, reactions during the formation of the solar system have to be considered to explain the $\mathrm{D}$ enrichment of the organics in meteorites. Two kinds of reactions could be involved in the synthesis of organics in chondrites: reactions at thermodynamic equilibrium between neutral species occurring in the disk mid plane, at relatively high temperature $(\mathrm{T}=400 \mathrm{~K}$, Fegley 1999) and ion/molecule 
or gas/grain reactions occurring at lower temperature $(\mathrm{T}<100 \mathrm{~K})$ but under UV or X-ray irradiation (Markwick et al. 2002). Reactions involving ions or interactions between gas and grains are kinetically favored at low temperature and are believed to induce large D-enrichment in organic molecules (Sandford et al. 2001; Willacy \& Woods 2009). In contrast, thermodynamic equilibrium in the warm midplane could theoretically induce only a modest D-enrichment in organic matter (Lécluse \& Robert 1994) with a D/H $<90 \times 10^{-6}$ in $\mathrm{CH}_{4}$, considering a starting $\mathrm{D} / \mathrm{H}$ of the protosolar nebula at $25 \times 10^{-6}$. These reactions would be kinetically inhibited (Hayatsu \& Anders 1981) but catalytic processes on grain surfaces like Fischer-Tropsch type reactions (FTT) have been proposed to produce a significant amount of organics under the assumed conditions of the inner protosolar disk (Studier et al. 1972; Nuth et al. 2008). The D/H of most chondrites IOMs, being much higher than $90 \times 10^{-6}$, can only be acquired in cold and ionized environments, by ion/molecule reactions for instance. Only the lowest D/H could account for the occurrence of thermodynamic reactions.

The interpretations of the large heterogeneities in $\mathrm{D} / \mathrm{H}$ at different scales are also debated. The simplest hypothesis would be that a common organic precursor, with a $\mathrm{D} / \mathrm{H}$ signature related to its environment of synthesis, would have evolved under specific conditions of each parent body (Alexander et al. 2010), leading to the variation of $\mathrm{D} / \mathrm{H}$ among the different classes as the result of isotopic exchange with fluids, loss of some organic reservoirs or reactions involving $H$ transfer. In such a scenario, D-rich hot spots would represent remnant grains of interstellar D-rich organic material that would not be affected by parent body processes (Busemann et al. 2006; Nakamura-Messenger et al. 2006). However, another hypothesis (Gourier et al. 2008; Remusat et al. 2009) suggests that the D-rich hot spots correspond to radical rich regions as organic radicals are D-rich and are heterogeneously distributed. As the radicals may have formed in the protosolar nebula, it would imply a local origin (i.e. not interstellar) for the D-rich hot spots. Then, if we can produce a large range of $\mathrm{D} / \mathrm{H}$ in organics within the protosolar nebula, or the parent molecular cloud, a pre-accretion diversity in isotopic composition of organic precursors could also explain the multiscale diversity in chondrites (Remusat et al. 2010). It must be noted that the most D-rich organic grains in chondrites does not show significant isotopic exchange with the mineral matrix, questioning a parent body effect to account for the observed diversity (Remusat et al. 2010). This assumption tackles the concept of a common precursor for all the chondrites and it implies that we should rather consider the organic matter in each chondrite group as a sampling of a range of isotopically and chemically distinct organic precursors. This diversity would reflect heterogeneities in the protosolar nebula or the parent molecular cloud: variations in ionization degree resulting in variations in the isotopic effect of ion/molecule reaction, different proportion of water/organics in ices depending on the location in respect to the snow line position, etc.. Thanks to turbulence in the disk (Ciesla 2009) organic particles could be exposed to very diverse environments and conditions before gathering at the location of the chondrites formations resulting in the association of very different organic grains in the same parent body. 


\section{Conclusion}

Many issues remain opened in the understanding of the volatile content of extraterrestrial objects and laboratory astrophysics could help to understand the message recorded in chondrites.

Organic matter occurs in association with low temperature components in chondrites. The molecular characterization of the organic molecules in meteorites allows us to compare them with organic matter detected in the interstellar medium and in molecular clouds. This may fill the gap between the understanding of interstellar reactions and the extraterrestrial organic compounds brought to laboratories by meteorites and comets. It may also reveal the processes that formed the organic compounds available in the Solar System before life started on Earth. Noble gases history seems related to organics origin in meteorites. Among all the questions around the noble gases content and properties, the processes that trapped them in meteorites remain unconstrained. Laboratory experiments are required to better understand how meteorites have acquired their noble gases content.

Organic matter is characterized by a high and heterogeneous deuterium content. This heterogeneity cannot be fully explained by parent body effects and indicates that it is a mixing of components with several origins and history. Cold chemistry seems to be recorded in their $\mathrm{D} / \mathrm{H}$ ratio, but the physical conditions (location, temperature, irradiation, etc.) still needs to be constrained. This is required in order to build a self-consistent model that would explain the formation and the evolution of organics in the solar system.

\section{References}

Alexander, C.M.O.D., Newsome, S.D., Fogel, M.L., et al., 2010, Geochim. Cosmochim. Acta, 74, 4417

Alpern, B., \& Benkheiri, Y., 1973, Earth Planet. Sci. Lett., 19, 422

Amelin, Y., Krot, A.N., Hutcheon, I.D., \& Ulyanov, A.A., 2002, Science, 297, 1678

Anders, E., \& Grevesse, N., 1989, Geochim. Cosmochim. Acta, 53, 197

Binet, L., Gourier, D., Derenne, S., \& Robert, F., 2002, Geochim. Cosmochim. Acta, 66, 4177

Binet, L., Gourier, D., Derenne, S., Robert, F., \& Ciofini, I., 2004, Geochim. Cosmochim. Acta, 68, 881

Bischoff, A., 1998, Meteorit. Planet. Sci., 33, 1113

Bonal, L., Quirico, E., Bourot-Denise, M., \& Montagnac, G., 2006, Geochim. Cosmochim. Acta, 70, 1849

Brearley, A.J., Heinrich, D.H., \& Karl, K.T., 2003, in Treatise on Geochemistry (Oxford: Pergamon), 247

Brearley, A.J., 2006, in Meteorites and the Early Solar System II, ed. D. Lauretta \&

H.Y.J.J. McSween (Tucson, USA: University of Arizona Press), 584

Bunch, T.E., \& Chang, S., 1980, Geochim. Cosmochim. Acta, 44, 1543

Busemann, H., Baur, H., \& Wieler, R., 2000, Meteorit. Planet. Sci., 35, 949 
Busemann, H., Young, A.F., Alexander, C.M.O.D., et al., 2006, Science, 312, 727

Ciesla, F.J., 2009, Icarus, 200, 655

Cooper, G.W., \& Cronin, J.R., 1995, Geochim. Cosmochim. Acta, 59, 1003

Cooper, G.W., Kimmich, N., Belisle, W., et al., 2001, Nature, 414, 879

Dartois, E., Muoz Caro, G.M., Deboffle, D., Montagnac, G., \& D'Hendecourt, L., 2005, A\&A, 432, 895

Derenne, S., \& Robert, F., 2010, Meteorit. Planet. Sci., 45, 1461

DuFresne, E.R., \& Anders, E., 1962, Geochim. Cosmochim. Acta, 26, 1085

Eiler, J.M., \& Kitchen, N., 2004, Geochim. Cosmochim. Acta, 68, 1395

Engel, M.H., \& Nagy, B., 1982, Nature, 296, 837

Fegley, B., 1999, Sp. Sci. Rev., 90, 239

Garvie, L.A.J., \& Buseck, P.R., 2004, Earth Planet. Sci. Lett., 224, 431

Geiss, J., \& Gloecker, G., 1998, Sp. Sci. Rev., 84, 239

Gilmour, I., Heinrich, D.H., \& Karl, K.T., 2003, in Treatise on Geochemistry, 269

Glavin, D.P., \& Dworkin, J.P., 2009, PNAS, 106, 5487

Gourier, D., Robert, F., Delpoux, O., et al., 2008, Geochim. Cosmochim. Acta, 72, 1914

Hartogh, P., et al., 2011, Nature, 478, 218

Hayatsu, R., Studier, M.H., Moore, L.P., \& Anders, E., 1975, Geochim. Cosmochim. Acta, 39, 471

Hayatsu, R., \& Anders, E., 1981, Topics Curr. Chem., 99, 1

Huang, Y., Alexandre, M.R., \& Wang, Y., 2007, Earth Planet. Sci. Lett., 259, 517

Huss, G.R., Lewis, R.S., \& Hemkin, S., 1996, Geochim. Cosmochim. Acta, 60, 3311

Kallemeyn, G.W., \& Wasson, J.T., 1981, Geochim. Cosmochim. Acta, 45, 1217

Kvenvolden, K.A., Lawless, J., Pering, K., et al., 1970, Nature, 228, 923

Krishnamurthy, R.V., Epstein, S., Cronin, J.R., Pizzarello, S., \& Yuen, G.U., 1992, Geochim. Cosmochim. Acta, 56, 4045

Lécluse, C., \& Robert, F., 1994, Geochim. Cosmochim. Acta, 58, 2927

Lewis, R.S., Srinivasan, B., \& Anders, E., 1975, Science, 190, 1251

Lodders, K., 2003, ApJ, 591, 1220

Markwick, A.J., Ilgner, M., Millar, T.J., \& Henning, T., 2002, A\&A, 385, 632

Marrocchi, Y., Derenne, S., Marty, B., \& Robert, F., 2005, Earth Planet. Sci. Lett., 236, 569

Marrocchi, Y., Marty, B., Reinhardt, P., \& Robert, F., 2011, Geochim. Cosmochim. Acta, 75, 6255

Millar, T.J., 2003, in Solar system history from isotopic signatures of volatile elements, ed. R. Kallenbach, T. Encrenaz \& J. Geiss et al. (ISSI), 73

Muoz Caro, G.M., et al., 2006, A\&A, 459, 147

Nakamura, K., Zolensky, M.E., Tomita, S., Nakashima, S., \& Tomeoka, K., 2002, Intern. J. Astrobio., 1, 179

Nakamura-Messenger, K., Messenger, S., Keller, L.P., Clemett, S.J., \& Zolensky, M.E., 2006, Science, 314, 1439

Nuth III, J.A., Johnson, N.M., \& Manning, S., 2008, ApJ, 673, L225

Orthous-Daunay, F.R., Quirico, E., Lemelle, L., et al., 2010, Earth Planet. Sci. Lett., 300,321 
Pearson, V.K., Sephton, M.A., Kearsley, A.T., et al., 2002, Meteorit. Planet. Sci., 37, 1829

Pendleton, Y.J., \& Allamandola, L.J., 2002, ApJS, 138, 75

Pizzarello, S., Cooper, G.W., \& Flynn, G.J., 2006, in Meteorites and the Early Solar System II, 625

Podosek, F.A., Heinrich, D.H., \& Karl, K.T., 2003, in Treatise on Geochemistry, 381

Remusat, L., Derenne, S., Robert, F., \& Knicker, H., 2005, Geochim. Cosmochim. Acta, 69, 3919

Remusat, L., Palhol, F., Robert, F., Derenne, S., \& France-Lanord, C., 2006, Earth Planet. Sci. Lett., 243, 15

Remusat, L., Le Guillou, C., Rouzaud, J.-N., et al., 2008, Meteorit. Planet. Sci., 43, 1099

Remusat, L., Robert, F., Meibom, A., et al., 2009, ApJ, 698, 2087

Remusat, L., Guan, Y., Wang, Y., \& Eiler, J.M., 2010, ApJ, 713, 1048

Robert, F., \& Epstein, S., 1982, Geochim. Cosmochim. Acta, 46, 81

Robert, F., 2002, Planet. Sp. Sci., 50, 1227

Robert, F., Derenne, S., Thomen, A., Anquetil, C., \& Hassouni, K., 2011, Geochim. Cosmochim. Acta, 75, 7522

Rouzaud, J.N., \& Oberlin, A., 1989, Carbon, 27, 517

Rubenstein, E., Bonner, W.A., Noyes, H.P., \& Brown, G.S., 1983, Nature, 306, 118

Saito, M., \& Kimura, Y., 2009, ApJ, 703, L147

Sandford, S., Bernstein, M.P., \& Dworkin, J.P., 2001, Meteorit. Planet. Sci., 36, 1117

Sephton, M.A., Pillinger, C.T., \& Gilmour, I., 1998, Geochim. Cosmochim. Acta, 62, 1821

Sessions, A.L., Sylva, S.P., Summons, R.E., \& Hayes, J.M., 2004, Geochim. Cosmochim. Acta, 68, 1545

Studier, M.H., Hayatsu, R., \& Anders, E., 1972, Geochim. Cosmochim. Acta, 36, 189

Wang, Y., Huang, Y., Alexander, C.M.O.D., Fogel, M., \& Cody, G., 2005, Geochim. Cosmochim. Acta, 69, 3711

Weisberg, M.K., McCoy, T.J., \& Krot, A.N., 2006, in Meteorites and the Early Solar System II, 19

Wieler, R., Busemann, H., \& Franchi, I.A., 2006, in Meteorites and the Early Solar System II, 499

Willacy, K., \& Woods, P.M., 2009, ApJ, 703, 479

Yabuta, H., Williams, L.B., Cody, G.D., Alexander, C.M.O.D., \& Pizzarello, S., 2007, Meteorit. Planet. Sci., 42, 37

Yang, J., \& Epstein, S., 1983, Geochim. Cosmochim. Acta, 47, 2199

Yuen, G.U., \& Kvenvolden, K.A., 1973, Nature, 246, 301 